\title{
Las competencias generales y transversales del Grado en Logopedia desde la perspectiva del alumno
}

\section{Generic and transversal competences in the speech-language pathology degree from the students' viewpoint}

\author{
Manuel Rodríguez Sánchez ${ }^{1}$ \\ manuerod@ucm.es \\ Pilar Revilla Rodríguez \\ previlla@ucm.es \\ Universidad Complutense de Madrid, España
}

\section{Resumen:}

Consecuentemente al valor curricular que se concede a la adquisición y desarrollo de competencias en el marco del Espacio Europeo de Educación Superior, este trabajo persigue un doble objetivo: analizar la valoración de la importancia que los alumnos de $4^{\circ}$ curso del Grado de Logopedia de la Universidad Complutense de Madrid conceden a las competencias generales y transversales descritas en el Título y, en segundo lugar, la percepción de su propia adquisición. Los resultados indican que la valoración que los alumnos otorgan a las competencias del Grado es, en todos los casos, alta y superior a la valoración del grado de desarrollo competencial que admiten haber adquirido, resultando diferencias estadísticamente significativas entre ambas percepciones.

\begin{abstract}
:
Resulting from the importance given to the acquisition and development of competences within the framework of the European Higher Education Area, this article pursues two goals: to analyze how $4^{\text {th }}$ year graduate students of Speech-Language Pathology in the Complutense University of Madrid assess the generic and transversal competences described in the title, and, secondly, how they perceive their own acquisition of these competences. Results indicate that in every case students give positive feedback about the degree's competences. Students' perceptions are in all cases higher than the actual competences they admit to have acquired, which gives rise to significant statistical differences between both views.
\end{abstract}

1 Dirección para correspondencia (correspondence address):

Manuel Rodríguez Sánchez. Dpto. de Didáctica y Organización Escolar. Facultad de Educación -Centro de formación del Profesorado-, Universidad Complutense de Madrid. C/ Rector Royo Villanova, s/n. Ciudad Universitaria - Campus de Moncloa. 28040 Madrid (España). 
Las competencias generales y transversales del Grado en Logopedia desde la perspectiva del alumno

Manuel Rodríguez Sánchez y Pilar Revilla Rodríguez

\section{Palabras clave:}

Competencias genéricas; competencias transversales; educación superior; Espacio Europeo de Educación Superior (EEES); logopedia.

\section{Key words:}

Generic competences; transversal competences; higher education; European Higher Education Area (EHEA); speechlanguage therapy.

\section{Résumé:}

À la vue de l'importance académique attribuée à l'acquisition et au développement des compétences dans le cadre de L'Espace Européen de L'Enseignement Supérieur, ce travail poursuit un double objectif. Le premier consiste à analyser l'importance pour les élèves de quatrième année d'Orthophonie de l'Université Complutense de Madrid, des différentes compétences, générales et transversales, de leur cursus. Le deuxième objectif prétend évaluer la perception personnelle du degré d'acquisition des compétences. Les résultats de l'étude révèlent que dans tous les cas, les élèves considèrent que les compétences obtenues, grâce au contenu académique de la Licence, sont relativement supérieures à celles réellement acquises et développées au niveau personnel. Les statistiques corroborent de manière significative les différences entre ces deux perceptions.

\section{Mots clés:}

Compétences génériques; compétences transférables; enseignement supérieur; Espace Européen de l'Enseignement Supérieur (EEES); orthophonie.

Fecha de recepción: 4-6-2014

Fecha de aceptación: 22-9-2015

\section{Introducción}

El curso 2012-2013 es el de la primera promoción de graduados en el marco del Espacio Europeo de Educación Superior (EEES). Son muchos los cambios que se ha producido, tanto en la definición de los títulos como en los aspectos metodológicos y en las actitudes de la comunidad universitaria. La adaptación de la Universidad española al marco del EEES ha traído consigo nuevas maneras de pensar y hacer en la educación superior centrando los desarrollos curriculares en la adquisición de determinadas competencias, en la potenciación del protagonismo del alumno en el proceso de enseñanza-aprendizaje, en el alejamiento de una forma de docencia basada casi exclusivamente en la clase magistral y en la búsqueda de metodologías docentes activas susceptibles de fomentar el desarrollo de las competencias pretendidas (RodríguezSánchez, 2011).

Si la formación por competencias es el núcleo sobre el que se ha hecho gravitar la reforma, la evaluación del éxito del nuevo enfoque pasa ineludiblemente por la determinación de las competencias adquiridas 
por los alumnos (López Ruiz, 2011). Como agentes principales en la construcción de su conocimiento y como sujetos de desarrollo competencial, conocer la valoración que los alumnos hacen de sus estudios y del grado competencial alcanzado es un elemento importante para conocer mejor los resultados del nuevo enfoque.

Este trabajo tiene como objetivos determinar, en primer lugar, la valoración de los alumnos sobre las competencias generales y transversales definidas para el Título de Grado en Logopedia en cuanto a la relevancia que les conceden y, en segundo lugar, determinar la autopercepción de su adquisición al finalizar los estudios. Así mismo, se han comparado ambas percepciones con el objeto de estimar si existen diferencias significativas entre la importancia concedida a las competencias y la percepción de su adquisición.

\section{Las competencias en el marco universitario}

En las últimas décadas se ha venido implantando en los sistemas educativos de los países desarrollados el enfoque de la educación por competencias y algo más tarde en España. Las razones que se invocan para el cambio de enfoque curricular son variadas, pero por lo general aluden a la complejidad que las sociedades modernas han adquirido en un proceso de globalización que no parece tener vuelta atrás y que exige del ciudadano ciertas habilidades y capacidades que le permitan desenvolverse en un mundo laboral complejo, tecnológico, competitivo y permanentemente cambiante. Desde esta lógica parece tener sentido el cambio de un enfoque centrado en objetivos educativos, orientados al desarrollo personal, por otro competencial centrado en el desarrollo de capacidades orientadas a la práctica laboral y al desarrollo profesional.

Las competencias se definen como la capacidad de los individuos para poner en marcha de manera integrada los conocimientos adquiridos y rasgos de personalidad que permiten resolver situaciones diversas en la vida profesional (Le Boterf, 2001). Desde una perspectiva semejante, Rey (1996) define las competencias como la capacidad de generar aplicaciones o soluciones adaptadas a la situación, movilizando los propios recursos y regulando el proceso hasta lograr la meta pretendida. Bunk (1994) define la competencia como el conjunto de conocimientos, de destrezas y de aptitudes que se precisan para ejercer una profesión, 
para resolver problemas profesionales de una manera autónoma y flexible y para colaborar en el contexto y en la organización laboral. Por su parte, Bisquerra (2004, p. 1) considera que "competencia es el conjunto de conocimientos, capacidades, habilidades y actitudes necesarias para realizar actividades diversas con un cierto nivel de calidad y eficacia". Según este autor, el dominio de una competencia permite producir un número infinito de acciones no programadas.

Para Valverde (2001) la competencia pone en igualdad de valor tanto las tareas como las cualidades personales que permiten realizarlas eficazmente en determinados contextos: "la competencia, así concebida, valora la capacidad del trabajador para poner en juego su saber adquirido en la experiencia. De esta forma, se entiende como una interacción dinámica entre distintos acervos de conocimientos, habilidades, destrezas, actitudes y aptitudes movilizados según las características del contexto y desempeño en que se encuentra el individuo" (p. 28).

Echeverría discrimina entre el saber necesario para afrontar determinadas situaciones y la capacidad de enfrentarse a las ellas. El saber está relacionado con la cualificación personal, pero el aprovechamiento de ésta depende del entorno estructural en donde pueda desarrollarla y de los ámbitos institucionales de la formación. En este sentido, posee competencia profesional "quien dispone de los conocimientos, destrezas y actitudes necesarias para ejercer su propia actividad laboral, resuelve los problemas de forma autónoma y creativa y está capacitado para colaborar con su entorno laboral y en la organización del trabajo" (Echeverría, 2001, p. 44). En el resumen ejecutivo preparado por la Organización para la Cooperación y el Desarrollo de Económico en el marco del Proyecto DeSeCo se indica también que "una competencia es más que conocimientos y destrezas. Involucra la habilidad de enfrentar demandas complejas, apoyándose en y movilizando recursos psicosociales (incluyendo destrezas y actitudes) en un contexto en particular" (OCDE, 2006, p. 3). En estas definiciones subyace como denominador común la idea de que la competencia supone la interacción entre las cualidades personales y las características del entorno, ante las que el sujeto competente es capaz de movilizar acciones y producir efectos deseables, intencionalmente previstos y regulados en su ejecución.

En el desarrollo del concepto se han identificado componentes, se han propuesto diversas clasificaciones y perfilado matices característicos de las competencias que pretenden hacerlo aceptable y operativo a las 
situaciones educativas y ámbitos profesionales en las que se aplica. Así, Echeverría (2002) desde un enfoque holístico del concepto y empleando un juego de palabras, concibe la competencia de acción profesional como "una cuestión de 'saber' -técnico y metodológico- y 'sabor' -participativo y personal-" (p. 17). Entiende este autor que la suma de estos cuatro componentes -saber, saber hacer, saber estar y saber ser, respectivamente- definen la estructura competencial que favorece que el sujeto pueda actuar con eficacia y eficiencia en los ámbitos profesionales. El saber técnico supone poseer conocimientos especializados y relacionados con determinado ámbito profesional, que permiten dominar como experto los contenidos y tareas acordes a su actividad laboral. El saber hacer metodológico supone saber aplicar los conocimientos a situaciones laborales concretas, utilizar procedimientos apropiados, solucionar problemas y transferir las experiencias adquiridas a situaciones nuevas. El saber estar participativo se refiere a estar atento a la evolución del mercado laboral, predispuesto al entendimiento interpersonal, a la comunicación y cooperación con los demás y, por último, el saber ser personal significa tener una imagen realista de sí mismo, asumir responsabilidades, tomar decisiones y relativizar frustraciones (Echeverría, 2002).

Barnett (2001) clasifica las competencias según dos vectores: uno que se dirige del mundo académico al mundo del trabajo y otro que marcha desde lo específico a lo general. En este esquema, Barnett identifica cuatro tipo de competencias: las específicas para la disciplina que se enseña; las interdisciplinarias, entendidas como el resultado de la enseñanza; las específicas de la profesión, aquellas para las que preparan los títulos universitarios y, por último, las personales transferibles, aquellas competencias que son desarrolladas en diferentes profesiones y puestos de trabajo y que denotan el grado de adaptabilidad de los profesionales al mundo laboral y social.

En el Proyecto Tunning (González y Wagenaar, 2003), se propone la clasificación de las competencias transversales en tres dimensiones o categorías: instrumentales, entendidas como más cognitivas y esenciales para el ejercicio de las profesiones; interpersonales, relacionadas con las capacidades de interactuar dinámicamente en equipos de trabajo, respetando las opiniones y la asignación de tareas y sistémicas, relacionadas con la capacidad de aprender, la autonomía e iniciativa personal o la creatividad. En los diferentes estudios consultados se ha podido constatar que no existe unidad de criterio a la hora de asignar las diferentes 
competencias a las categorías de clasificación que se han propuesto, ni tampoco se encuentra consenso en cuanto a las categorías a considerar. Ello es lógico, puesto que la propia definición de competencia presenta cierto grado de ambigüedad en el sentido de que, en sí misma, supone la reunión y síntesis de capacidades cognitivas, instrumentales y personales que en su conjunto han de configurar el perfil del estudiante o del trabajador. Determinar en qué medida recae el peso mayor de una u otra capacidades no es en muchos casos tarea fácil y depende de la titulación en particular a la que se refiera.

Sobre la percepción de los estudiantes en torno a las competencias se han encontrado estudios en la línea del presente realizados en otras titulaciones y universidades españolas y extranjeras. El estudio de Cebriá i Iranzo, Díaz Díaz, y Igual Camacho (2009) recoge la opinión que los estudiantes de fisioterapia de la Universidad de Valencia tienen sobre la importancia y el grado de formación recibido en las competencias recogidas en el Libro Blanco del titulo de grado, resultando que los estudiantes valoraron en mayor medida las competencias relacionadas con el conocimiento y el ejercicio de la profesión y no tanto con las relacionadas con la administración y organización de la práctica y los conocimientos relacionados con el contexto sociopolítico de la atención, coincidentes con los aspectos en los que se reconocían mejor formados.

De la Iglesia Villasol realizó un estudio descriptivo de los resultados de la encuesta realizada a los estudiantes de cuarto y quinto curso de las licenciaturas de Economía y Administración de Empresas, en las Facultades de Ciencias Económicas y Empresariales de la Universidad Complutense de Madrid y de la Universidad de Alcalá de Henares, sobre la percepción que ellos tienen de su propio grado de desarrollo competencial y la valoración que hacen de la relevancia de dichas competencias para el ejercicio profesional, concluyendo que la importancia que conceden a las distintas competencias en el trabajo es mayor que el grado de desarrollo competencial que declaran tener (De la Iglesia Villasol, 2011); (De la Iglesia Villasol, 2012).

El estudio de Díez González et al. (2009), sobre metodologías docentes y desarrollo de competencias ante la adaptación y armonización al EEES en las titulaciones de Maestro en diferentes especialidades y Psicopedagogía de la Universidad de Valladolid, los resultados mostraron que los alumnos percibían que su nivel de competencias era mejor en los primeros cursos y que disminuían gradualmente hacia cursos superiores. 
Montoro-Sánchez, Mora-Valentín, y Ortiz-de-Urbina-Criado (2012) realizaron un estudio que identificó las competencias más demandadas por los empresarios en el mercado laboral para los alumnos con estudios de Dirección de Empresas y llevaron a cabo un análisis comparativo entre las competencias requeridas por las empresas (competencias prácticas) y las competencias disponibles en los alumnos como resultado de su formación (competencias teóricas). Los resultados indicaron que en la mayoría de los casos hay una clara correlación entre las competencias requeridas y las disponibles en el alumno, si bien hay casos en los que se encontraron diferencias.

El trabajo de Romero Cerezo, Zagalaz Sánchez, Romero Rodríguez, y Martínez López (2011) pretende determinar las competencias profesionales más valoradas por los estudiantes de la Titulación de Maestro en Educación Física para su posterior desempeño laboral en las universidades de Granada, Jaén y Almería. En sus conclusiones, los autores destacan como competencias más relevantes en la opinión de los alumnos la capacidad para trabajar en equipo, la capacidad para estimular y transmitir valores y la capacidad de establecer la relación necesaria entre la actividad física con los hábitos de higiene, posturales y de salud.

El presente estudio plantea como objetivos conocer la importancia que los alumnos de $4^{\circ}$ curso de Grado en Logopedia otorgan a las competencias transversales y generales descritas en el Título de Grado y su percepción del grado de adquisición competencial que han obtenido. No hay constancia de estudios sobre las percepciones de los alumnos sobre las competencias en la titulación que nos ocupa, por lo que se ha considerado de interés conocer la valoración de los alumnos del Grado de Logopedia de la Universidad Complutense de Madrid para la orientación de la mejora de la acción docente y de la calidad del título.

\section{Metodología}

El marco de referencia para llevar a cabo este estudio es el Libro Blanco del Titulo de Grado en Logopedia (Gallego, 2004). Allí se realiza un estudio pormenorizado de las competencias que el futuro graduado en logopedia debería poseer, valorándose un listado de competencias mediante un estudio de campo en el que fueron consultados profesionales, empleadores y docentes en Logopedia, abriéndose posteriormente un 
debate entre los representantes de las universidades. El estudio también concluyó que las diferencias en cuanto a cuál debiera ser el perfil del logopeda y cuál debería ser su marco de trabajo fueron muy escasas entre los participantes, lo que permitió abordar el diseño del título desde la constancia de un alto nivel de consenso entre profesionales, empleadores y docentes universitarios.

Las competencias transversales son comunes a la mayoría de las titulaciones universitarias aunque, lógicamente, han de particularizarse a las características y contextos propios de cada titulación. Se relacionan con la puesta en práctica integrada de aptitudes, rasgos de personalidad, conocimientos adquiridos y también valores (Cela, Arias, y Esteve, 2009). Las competencias generales se refieren a aspectos genéricos, pero relacionados directamente con el ejercicio de la profesión. En el caso que nos ocupa implican el desarrollo de habilidades para la evaluación, diagnóstico e intervención en las alteraciones del lenguaje que orientan la práctica logopédica desde planteamientos formativos teóricos, científicos, prácticos y tecnológicos, como se muestra en la Tabla 1.

El marco propio para el aprendizaje de este tipo de competencias es el entorno laboral, por lo que su abordaje en la universidad es una tarea compleja que requiere la introducción de metodologías docentes activas e innovadoras, entornos de simulación de situaciones profesionales y prácticas reales ajustadas a las futuras tareas profesionales, todo ello propiciado por cambios en las actitudes de los docentes y de los alumnos. Una parte importante de la formación universitaria tiene un carácter profesionalizador, como es el caso de la formación de logopedas, cuyo Grado tiene características directamente orientadas al ejercicio profesional.

Tabla 1. Competencias transversales y generales del Grado de Logopedia (CT: Competencia Transversal, CG: Competencia General). COMPETENCIAS

CT1 Ser capaz de desarrollar habilidades como: regular su propio aprendizaje, resolver problemas, razonar críticamente y adaptarse a situaciones nuevas.

CT2 Comunicar de manera oral y escrita sus observaciones y conclusiones al paciente, a sus familiares y al resto de profesionales que intervienen en su atención adaptándose a las características sociolingüísticas del entorno.

CT3 Presentar una adecuada producción del habla, estructuración del lenguaje y calidad de la voz. 
CT4 Manejar las tecnologías de la comunicación y la información.

CT5 Observar y escuchar activamente.

CT6 Trabajar en equipo.

CT7 Demostrar capacidad de organización y planificación.

CT8 Demostrar capacidad de análisis y síntesis.

CG1 Diseñar, implementar y evaluar acciones de prevención de los trastornos de la comunicación y el lenguaje.

CG2 Explorar, evaluar, diagnosticar y emitir pronóstico de evolución de los trastornos de la comunicación y el lenguaje desde una perspectiva multidisciplinar.

CG3 Usar las técnicas e instrumentos de exploración propios de la profesión y registrar, sintetizar e interpretar los datos aportados integrándolos en el conjunto de la información.

CG4 Dominar la terminología que permita interactuar eficazmente con otros profesionales.

CG5 Diseñar y llevar a cabo los tratamientos logopédicos, tanto individuales como colectivos, estableciendo objetivos y etapas, con los métodos, técnicas y recursos más eficaces y adecuados, y atendiendo a las diferentes etapas evolutivas del ser humano.

CG6 Seleccionar, implementar y facilitar el aprendizaje de sistemas de comunicación aumentativos, así como el diseño y uso de prótesis y ayudas técnicas necesarias, adaptados a las condiciones físicas, psicológicas y sociales de sus pacientes.

CG7 Asesorar a familias y al entorno social de los usuarios, favoreciendo su participación y colaboración en el tratamiento logopédico.

CG8 Trabajar en los entornos escolar, asistencial y sanitario formando parte del equipo profesional. Asesorar en la elaboración, ejecución de políticas de atención y educación sobre temas relacionados con Logopedia.

CG9 Comprender y valorar las producciones científicas que sustentan el desarrollo profesional del logopeda (competencia no incluida en el cuestionario).

CG10 Conocer los límites de sus competencias y saber identificar si es necesario un tratamiento interdisciplinar.

CG11 Explicar y argumentar el tratamiento seleccionado.

CG12 Fomentar las habilidades comunicativas en la población general.

CG13 Conocer y ser capaz de integrar los fundamentos biológicos (anatomía y fisiología), psicológicos (procesos y desarrollo evolutivo), lingüísticos y pedagógicos de la intervención logopédica en la comunicación, el lenguaje, el habla, la audición, la voz y las funciones orales no verbales. 
Las competencias generales y transversales del Grado en Logopedia desde la perspectiva del alumno

Manuel Rodríguez Sánchez y Pilar Revilla Rodríguez

CG14. Conocer los trastornos de la comunicación, el lenguaje, el habla, la audición, la voz y las funciones orales no verbales.

CG15 Conocer y valorar de forma crítica las técnicas y los instrumentos de evaluación y diagnóstico en Logopedia, así como los procedimientos de la intervención logopédica.

CG16 Conocer y valorar de forma crítica la terminología y la metodología propias de la investigación logopédica.

CG17 Elaborar y redactar informes de exploración y diagnóstico, seguimiento, finalización y derivación.

\section{Participantes}

En este estudio interesaba conocer las percepciones de los alumnos que estuvieran a punto de concluir sus estudios de grado, considerando la oportunidad de representar a la primera promoción del título. Por ello la encuesta se dirigió a la totalidad de los 75 alumnos matriculados en $4^{\circ}$ curso del Grado de Logopedia. De los 75 cuestionarios distribuidos se han recogido 48, lo que supone una tasa de respuesta del 64\%. Al nivel de confianza de 95\% el intervalo de confianza es del 8,5\%.

\section{Instrumento}

Se ha empleado un cuestionario de 28 ítems correspondientes a las competencias definidas para el Título de Grado en Logopedia (Tabla 1), y que encuentran sus referentes en Gallego (2004), evaluadas por una escala Likert de cinco valores en la que cada competencia se valora desde 1 como muy poco importante, si se trata de la relevancia de la competencia o muy baja, si se trata de la percepción de su adquisición, hasta 5, que corresponde a muy importante o muy alta, respectivamente. El coeficiente de fiabilidad alfa de Cronbach indica que el instrumento posee suficiente consistencia interna $(\alpha=.892)$. El cuestionario se aplicó a los alumnos en la última semana del mes de mayo del curso 2012-13.

\section{Procedimiento}

En este estudio las competencias se han agrupado en tres dimensiones: cognitiva, técnico-profesional y personal, atendiendo a su carácter predominante: conocimientos teóricos, conocimientos procedimentales aplicados a la práctica de la profesión y actitudes personales y valores 
que debe poseer el logopeda. En la Tabla 2 se ofrece esta categorización, observándose que la dimensión técnico-profesional es la que contiene mayor número de competencias, tanto transversales como generales. Por otra parte, no se ha podido determinar ninguna competencia general cuyo peso recayera claramente en la dimensión personal, lo que es coherente con el perfil declarado en la definición del título de Grado en Logopedia, en el que predomina una orientación eminentemente clínica y encaminada a la práctica profesional que se resume en las siguientes capacidades:

- Prevenir, detectar, identificar, evaluar, y proporcionar tratamiento e intervención a personas de todas las edades con, o en riesgo de sufrir las patologías o trastornos que son objeto de su actuación.

- Aconsejar a los individuos con estos trastornos, así como a sus familias, cuidadores u otros profesionales, y actuar como testigo experto o perito, si es requerido para ello.

- Seleccionar, prescribir, dispensar y proporcionar asistencia para apoyar el uso efectivo de recursos de comunicación aumentativa y alternativa, así como prótesis de comunicación y otros dispositivos de ayuda.

- Enseñar, supervisar, y dirigir programas clínicos y educativos, así como desarrollar y supervisar programas y actividades de investigación relacionadas con el lenguaje, la comunicación, y sus trastornos.

- Evaluar la eficacia de su actividad, modificar los servicios en relación con sus evaluaciones y difundir estos hallazgos.

- Proporcionar servicios en contextos que se juzgan apropiados, incluyendo pero no limitándose al cuidado de la salud, como en los marcos educativo, comunitario, vocacional y del hogar.

- Intervenir en los procesos de mejora de las habilidades comunicativas de personas en la población general, sin necesidad de que existan alteraciones o trastornos. 
Tabla 2. Agrupación por dimensiones de las competencias Generales y Transversales del Grado de Logopedia.

\begin{tabular}{lll}
\hline DIMENSIÓN & Competencias generales & Competencias transversales \\
\hline Cognitiva & CG13; CG14; CG15; CG16 CT7; CT8 \\
\hline & CG1; CG2; CG3; CG4; & \\
Técnico-profesional & CG5; CG6; CG7; CG8; & CT2; CT4 \\
& CG10; CG11; CG12; CG17 & \\
$\begin{array}{l}\text { Personal (actitudes y } \\
\text { valores) }\end{array}$ & CT1; CT3; CT5; CT6 \\
\hline
\end{tabular}

\section{Resultados}

En la Tabla 3 se muestra el resumen de los resultados obtenidos para cada una de las competencias, considerando las medias, las desviaciones típicas y las diferencias de medias como estadísticos calculados.

Valoración de Competencias Transversales. La mayor valoración en cuanto a su relevancia es CT2 (Comunicar de manera oral y escrita sus observaciones y conclusiones al paciente, a sus familiares y al resto de profesionales que intervienen en su atención adaptándose a las características sociolingüísticas del entorno, $\bar{X}=4.94, D T=.245)$, seguida de CT3 (Presentar una adecuada producción del habla, estructuración del lenguaje y calidad de la voz, $\bar{X}=4.88, D T=.334$ ). Las puntuaciones medias más bajas corresponden a las competencias CT4 (Manejar las tecnologías de la comunicación y la información, $\bar{X}=4.29$, DT=.771) y CT1A (Ser capaz de desarrollar habilidades como: regular su propio aprendizaje, $\bar{X}=4.35, D T=.699$ ).

Valoración de Competencias Generales. CG5 (Diseñar y llevar a cabo tratamientos) y CG17 (Elaborar y redactar informes) son las que han obtenido mayor reconocimiento de su relevancia, prácticamente el máximo valor de la escala, coincidiendo la media y la desviación para ambas $(\bar{X}=4.98, D T=.144)$, seguidas de CG14 (Conocer los trastornos, $\bar{X}=4.94$, $D T=.320$ ), CG2 (Explorar, evaluar, diagnosticar y pronosticar, $\bar{X}=4.92$, $D T=.279)$ y CG13 (Conocer e integrar los fundamentos biológicos, psicológicos, lingüísticos y pedagógicos, $\bar{X}=4.92, D T=.279$ ). Por el contrario, la competencia menos valorada, aunque aún dentro del rango alto de puntuación, fue CG12 (Fomentar las habilidades comunicativas de la población, $\bar{X}=4.00, D T=.923$ ) (Tabla 3).

Percepción de adquisición de Competencias Transversales. La com- 
petencia transversal que los alumnos consideran adquirida en mayor grado es CT6 (Trabajo en equipo, $\bar{X}=4.40, D T=.676$ ), seguida de CT5 (Observación y escucha activas, $\bar{X}=4.02, D T=.785$ ). Las restantes competencias transversales se consideran aceptablemente adquiridas, con medias comprendidas entre 3.10 y 3.63, a excepción de CT3 (Comunicación oral y escrita, $\bar{X}=2.92, D T=1.145$ ) y CT4 (TIC, $\bar{X}=2.83, D T=1.136)$, que es la que peor puntuación obtiene.

Percepción de adquisición de Competencias Generales. El grado de adquisición de las competencias generales en opinión de los alumnos es asimismo bajo. La competencia general que mayor valoración recibe es CG14 (Conocer los trastornos, $\bar{X}=4.29, D T=.713$ ). Las restantes competencias generales se consideran adquiridas en menor medida, con medias que oscilan entre puntuaciones por debajo del valor central de la escala hasta valores próximos a 4 . Valores inferiores al valor central son los asignados a CG1 (Diseño, implementación y evaluación de acciones de prevención, $\bar{X}=2.98, D T=.812$ ), CG12 (Fomentar las habilidades comunicativas de la población, $\bar{X}=2.98, D T=1.021$ ), CG16 (Conocer y valorar críticamente la terminología y la metodología propias de la investigación logopédica, $\bar{X}=2.98, D T=.911$ ), CG5 (Diseñar y llevar a cabo tratamientos, $\bar{X}=2.88, D T=1.003$ ), CG8 (Trabajar en entornos escolares, asistencial y sanitario, $\bar{X}=2.73, D T=1.047$ ) y CG17 (Elaborar y redactar informes, $\bar{X}=2.60, D T=1.067)$, que es la que peor consideración de adquisición recibe.

Diferencias entre relevancia y adquisición. Las valoraciones de adquisición de competencias son sensiblemente inferiores en relación a las otorgadas a su relevancia en todos los casos, como lo ponen de manifiesto las diferencias de medias (Tabla 3). Las menores diferencias, inferiores a un grado de la escala, se observan en CT6 (Trabajo en equipo, 0.25); CT5 (Observación y escucha activas. 0.69); CG14 (Conocer los trastornos, 0.65); CT1A (Regular el propio aprendizaje, 0.77); CT7 (Organización y planificación, 0.87); CT8 (Análisis y síntesis, 0.98) y CG4 (Dominar la terminología para interactuar con otros profesionales, 0.85). Las diferencias mayores observadas, iguales o superiores o dos grados de la escala, corresponden a CG17 (Elaborar y redactar informes, 2.38); CG5 (Diseñar y llevar a cabo tratamientos, 2.10); CG8 (Trabajar en entornos escolares, asistenciales y sanitarios, 2.00) y CT2 (Comunicación oral y escrita, 2.02). 
Las competencias generales y transversales del Grado en Logopedia desde la perspectiva del alumno

Manuel Rodríguez Sánchez y Pilar Revilla Rodríguez

Tabla 3. Estadísticos descriptivos.

\begin{tabular}{|c|c|c|c|c|}
\hline Competencias & & Medias & Desv. típ. & Dif. \\
\hline \multirow{2}{*}{ CT1A Regular el propio aprendizaje } & REL & 4,35 & 699 & \multirow[b]{2}{*}{0,77} \\
\hline & ADQ & 3,58 & ,846 & \\
\hline \multirow{2}{*}{ CT1B Resolver problemas } & REL & 4,60 &, 536 & \multirow[b]{2}{*}{1,41} \\
\hline & $\overline{A D Q}$ & 3,19 & ,734 & \\
\hline \multirow{2}{*}{ CT1C Razonar críticamente } & REL & 4,60 &, 644 & \multirow[b]{2}{*}{1,31} \\
\hline & ADQ & 3,29 & 922 & \\
\hline \multirow[b]{2}{*}{ CT1D Adapt. a situaciones nuevas } & REL & 4,60 &, 536 & \multirow[b]{2}{*}{1,10} \\
\hline & ADQ & 3,50 & 1,052 & \\
\hline \multirow{2}{*}{ CT2 Comunicación oral y escrita } & REL & 4,94 & ,245 & \multirow[b]{2}{*}{2,02} \\
\hline & $\overline{A D Q}$ & 2,92 & 1,145 & \\
\hline \multirow{2}{*}{ CT3 Producción del habla } & REL & 4,88 & ,334 & \multirow[b]{2}{*}{1,78} \\
\hline & ADQ & 3,10 & 1,189 & \\
\hline \multirow{2}{*}{ CT4 TIC } & REL & 4,29 & 771 & \multirow[b]{2}{*}{1,46} \\
\hline & ADQ & 2,83 & 1,136 & \\
\hline \multirow{2}{*}{ CT5 Observación y escucha activas } & REL & 4,71 &, 544 & \multirow[b]{2}{*}{0,69} \\
\hline & ADQ & 4,02 & ,785 & \\
\hline \multirow{2}{*}{ CT6 Trabajo en equipo } & REL & 4,65 & ,526 & \multirow[b]{2}{*}{0,25} \\
\hline & $\overline{A D Q}$ & 4,40 &, 676 & \\
\hline \multirow{2}{*}{ CT7 Organización y planificación } & REL & 4,50 & ,715 & \multirow[b]{2}{*}{0,87} \\
\hline & $\overline{\mathrm{ADQ}}$ & 3,63 & 1,044 & \\
\hline \multirow{2}{*}{ CT8 Análisis y síntesis } & REL & 4,46 & 683 & \multirow[b]{2}{*}{0,98} \\
\hline & ADQ & 3,48 & 967 & \\
\hline \multirow{2}{*}{$\begin{array}{l}\text { CG1 Diseño, implementación y eval. de } \\
\text { acciones de prevención }\end{array}$} & REL & 4,88 & ,334 & \multirow[b]{2}{*}{1,90} \\
\hline & $\overline{\mathrm{ADQ}}$ & 2,98 & 812 & \\
\hline \multirow{2}{*}{$\begin{array}{l}\text { CG2 Explorar, evaluar, diagnosticar y } \\
\text { pronosticar }\end{array}$} & REL & 4,92 & 279 & \multirow[b]{2}{*}{1,86} \\
\hline & $\overline{\mathrm{ADQ}}$ & 3,06 & 976 & \\
\hline \multirow{2}{*}{ CG3 Uso de técnicas e instrumentos } & REL & 4,88 & ,393 & \\
\hline & ADQ & 3,08 & 846 & 1,80 \\
\hline CG4 Dominar la terminología para inte- & REL & 4,81 & ,491 & \\
\hline ractuar con otros profesionales & $\overline{\mathrm{ADQ}}$ & 3,96 & ,798 & 0,85 \\
\hline & REL & 4,98 & , 144 & \\
\hline CG5 Diseñar y llevar a cabo tratamientos & $\overline{A D Q}$ & 2,88 & 1,003 & 2,10 \\
\hline$\Delta C$ & REL & 4,77 & 472 & \\
\hline$c$ & ADQ & 3,23 & 1,016 & 1,54 \\
\hline CG7 Asesorar a las familias y entornos & REL & 4,77 & 472 & \\
\hline sociales & $\overline{A D Q}$ & 3,25 & 1,021 & 1,52 \\
\hline CG8 Trabajar en entornos escolares, asis- & REL & 4,73 & 494 & \\
\hline tencial y sanitario & ADQ & 2,73 & 1,047 & 2,00 \\
\hline
\end{tabular}




\begin{tabular}{|c|c|c|c|c|}
\hline Competencias & & Medias & Desv. típ. & Dif. \\
\hline \multirow{2}{*}{$\begin{array}{l}\text { CG10 Conocer las competencias profesio- } \\
\text { nales }\end{array}$} & REL & 4,73 & 494 & \\
\hline & $\overline{\mathrm{ADQ}}$ & 3,56 & 1,183 & 1,17 \\
\hline \multirow{2}{*}{$\begin{array}{l}\text { CG11 Explicar y argumentar los tratamien- } \\
\text { tos seleccionados }\end{array}$} & REL & 4,83 & ,429 & \\
\hline & ADQ & 3,42 & 1,048 & 1,41 \\
\hline \multirow{2}{*}{$\begin{array}{l}\text { CG12 Fomentar las habilidades comunica- } \\
\text { tivas de la población }\end{array}$} & REL & 4,00 & 923 & \\
\hline & $\overline{\mathrm{ADQ}}$ & 2,98 & 1,021 & 1,02 \\
\hline \multirow{2}{*}{$\begin{array}{l}\text { CG13 Conocer e integrar los fundamentos } \\
\text { biológicos, psicológicos, lingüísticos y } \\
\text { pedagógicos }\end{array}$} & REL & 4,92 &, 279 & \\
\hline & ADQ & 3,21 & 824 & 1,71 \\
\hline \multirow{2}{*}{ CG14 Conocer los trastornos } & REL & 4,94 & ,320 & \\
\hline & ADQ & 4,29 & ,713 & 0,65 \\
\hline \multirow{2}{*}{$\begin{array}{l}\text { CG15 Conocer y valorar críticamente } \\
\text { técnicas e instrumentos de evaluación y } \\
\text { diagnóstico }\end{array}$} & REL & 4,85 & 412 & \\
\hline & ADQ & 3,38 &, 890 & 1,47 \\
\hline \multirow{2}{*}{$\begin{array}{l}\text { CG16 Conocer y valorar críticamente la } \\
\text { terminología y la metodología propias de } \\
\text { la investigación log. }\end{array}$} & REL & 4,54 & 617 & \\
\hline & ADQ & 2,98 & ,911 & 1,56 \\
\hline \multirow{2}{*}{ CG17 Elaborar y redactar informes } & REL & 4,98 &, 144 & \\
\hline & $\mathrm{ADQ}$ & 2,60 & 1,067 & 2,38 \\
\hline $\mathrm{N}$ válido (según lista) & 48 & & & \\
\hline
\end{tabular}

El Gráfico1 refleja de manera sintética las diferencias encontradas. Cabe destacar que en todas las competencias los alumnos reconocen una menor adquisición en relación a la importancia, existiendo menores diferencias en las competencias referidas a la metodología de trabajo y, en menor medida, a los conocimientos teóricos adquiridos. 
Las competencias generales y transversales del Grado en Logopedia desde la perspectiva del alumno

Manuel Rodríguez Sánchez y Pilar Revilla Rodríguez

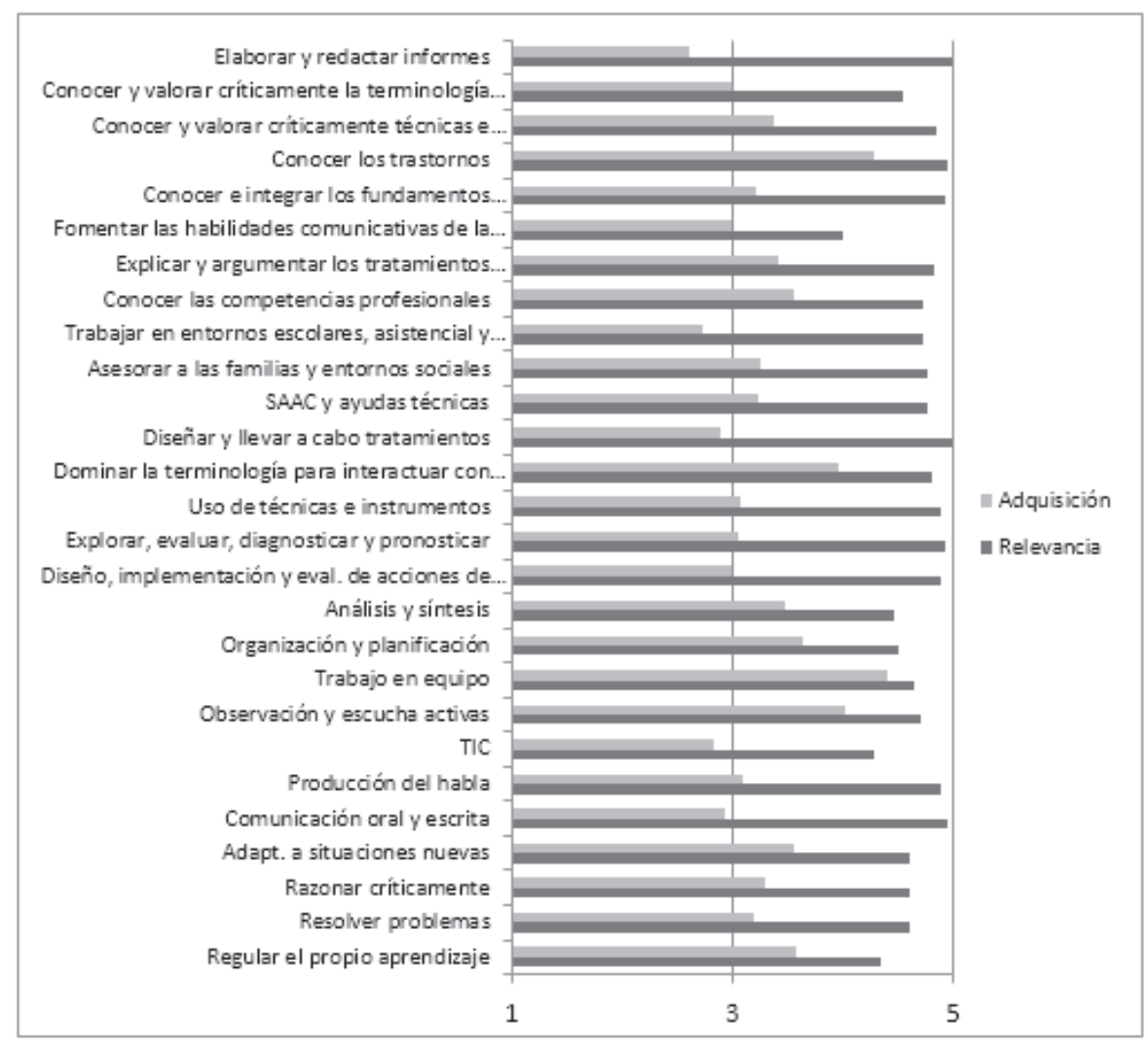

\section{Gráfico 1. Relevancia y adquisición de competencias. Gráfico com- parativo de medias.}

En tanto que las valoraciones resultan claramente divergentes entre el grado de importancia y el grado de adquisición, se procedió a efectuar pruebas no paramétricas de contraste de medias (Tabla 4), resultando la existencia de diferencias estadísticamente significativas al nivel de .01 en todas las competencias, exceptuando la competencia general CT6, trabajo en equipo. 
Tabla 4. Estadísticos de contraste.

\begin{tabular}{|c|c|c|c|}
\hline & $\begin{array}{l}\text { U de } \\
\text { Mann- } \\
\text { Whitney }\end{array}$ & $\begin{array}{cc}\text { W de } \\
\text { Wilcoxon }\end{array}$ & $\begin{array}{c}\text { Sig. } \\
\text { asintót. } \\
\text { (bilateral) }\end{array}$ \\
\hline -Regular el propio aprendizaje & 590,500 & $1766,500-4,344$ & , 000 \\
\hline -Resolver problemas & 187,500 & $1363,500-7,404$ & ,000 \\
\hline -Razonar críticamente & 274,500 & $1450,500-6,770$ & , 000 \\
\hline -Adapt. a situaciones nuevas & 420,000 & $1596,000-5,707$ & , 000 \\
\hline -Comunicación oral y escrita & 118,500 & $1294,500-8,178$ & , 000 \\
\hline -Producción del habla & 180,000 & $1356,000-7,643$ & , 000 \\
\hline$-\mathrm{TIC}$ & 362,000 & $1538,000-5,979$ &, 000 \\
\hline -Observación y escucha activas & 573,000 & $1749,000-4,692$ & , 000 \\
\hline -Trabajo en equipo & 924,000 & $2100,000-1,923$ &, 054 \\
\hline -Organización y planificación & 573,500 & $1749,500-4,501$ &, 000 \\
\hline -Análisis y síntesis & 499,500 & $1675,500-5,037$ &, 000 \\
\hline $\begin{array}{l}\text {-Diseño, implementación y eval. de ac- } \\
\text { ciones de prevención }\end{array}$ & 39,000 & $1215,000-8,627$ & ,000 \\
\hline -Explorar, evaluar, diagnosticar y pronosticar & 60,000 & $1236,000-8,533$ &, 000 \\
\hline -Uso de técnicas e instrumentos & 59,500 & $1235,500-8,508$ &, 000 \\
\hline $\begin{array}{l}\text {-Dominar la terminología para interactuar } \\
\text { con otros profesionales }\end{array}$ & 465,000 & $1641,000-5,634$ & , 000 \\
\hline -Diseñar y llevar a cabo tratamientos & 53,500 & $1229,500-8,732$ &, 000 \\
\hline -SAAC y ayudas técnicas & 209,000 & $1385,000-7,331$ &, 000 \\
\hline -Asesorar a las familias y entornos sociales & 214,000 & $1390,000-7,294$ & , 000 \\
\hline $\begin{array}{l}\text {-Trabajar en entornos escolares, asisten- } \\
\text { cial y sanitario }\end{array}$ & 139,000 & $1315,000-7,763$ & ,000 \\
\hline -Conocer las competencias profesionales & 446,500 & $1622,500-5,590$ &, 000 \\
\hline $\begin{array}{l}\text {-Explicar y argumentar los tratamientos } \\
\text { seleccionados }\end{array}$ & 287,500 & $1463,500-6,866$ & ,000 \\
\hline $\begin{array}{l}\text {-Fomentar las habilidades comunicativas } \\
\text { de la población }\end{array}$ & 555,000 & $1731,000-4,535$ &, 000 \\
\hline $\begin{array}{l}\text {-Conocer e integrar los fundamentos } \\
\text { biológicos, psicológicos, lingüísticos y } \\
\text { pedagógicos }\end{array}$ & 84,000 & $1260,000-8,386$ &, 000 \\
\hline -Conocer los trastornos & 558,500 & $1734,500-5,398$ & , 000 \\
\hline $\begin{array}{l}\text {-Conocer y valorar críticamente técnicas e } \\
\text { instrumentos de evaluación y diagnóstico }\end{array}$ & 216,500 & $1392,500-7,437$ & ,000 \\
\hline $\begin{array}{l}\text {-Conocer y valorar críticamente la termi- } \\
\text { nología y la metodología propias de la } \\
\text { investigación log. }\end{array}$ & 231,000 & $1407,000-7,011$ &, 000 \\
\hline -Elaborar y redactar informes & 5,000 & $1181,000-9,009$ &, 000 \\
\hline
\end{tabular}

Variable de agrupación: Importancia -Adquisición 
Se ha realizado así mismo un análisis por dimensiones (Tabla 2) con el fin de comparar las diferencias entre los valores medios globales. En la Tabla 5 se muestran las medias de las competencias asociadas a la dimensión cognitiva y los promedios globales. La media del conjunto de las competencias generales por reconocimiento de su importancia es muy próxima al valor máximo de la escala $(\bar{X}=4.81)$ y superior al de las competencias transversales $(\bar{X}=4.48)$, promedio que también se sitúa en la parte alta de la escala. Las valoraciones de la adquisición se aproximan más al valor central, $\bar{X}=3.47$ y $\bar{X}=3.56$ para las competencias generales y transversales, respectivamente.

Tabla 5. Promedios de la dimensión cognitiva.

\begin{tabular}{|c|c|c|c|c|}
\hline \multirow{2}{*}{\multicolumn{2}{|c|}{ Dimensión Competencias generales }} & Medias & \multirow{2}{*}{$\begin{array}{l}\text { Competencias } \\
\text { transversales }\end{array}$} & \multirow{2}{*}{$\begin{array}{l}\text { Medias } \\
\text { Rel. Adq. }\end{array}$} \\
\hline & & Rel. Adq. & & \\
\hline Cognitiva & $\begin{array}{l}\text { CG13 Conocer y ser capaz de } \\
\text { integrar los fundamentos bio- } \\
\text { lógicos (anatomía y fisiología), } \\
\text { psicológicos (procesos y de- } \\
\text { sarrollo evolutivo), lingüísticos } \\
\text { y pedagógicos de la interven- } \\
\text { ción logopédica en la comu- } \\
\text { nicación, el lenguaje, el habla, } \\
\text { la audición, la voz y las funcio- } \\
\text { nes orales no verbales. } \\
\text { CG14 Conocer los trastornos } \\
\text { de la comunicación, el lengua- } \\
\text { je, el habla, la audición, la voz } \\
\text { y las funciones orales no ver- } \\
\text { bales. } \\
\text { CG15 Conocer y valorar de } \\
\text { forma crítica las técnicas y los } \\
\text { instrumentos de evaluación y } \\
\text { diagnóstico en Logopedia, así } \\
\text { como los procedimientos de } \\
\text { la intervención logopédica. } \\
\text { CG16 Conocer y valorar de } \\
\text { forma crítica la terminología } \\
\text { y la metodología propias de la } \\
\text { investigación logopédica. }\end{array}$ & $4,853,38$ & $\begin{array}{l}\text { CT7 Demos- } \\
\text { trar capacidad } \\
\text { de organiza- } \\
\text { ción y planifi- } \\
\text { cación. } \\
\text { CT8 Demos- } \\
\text { trar capacidad } \\
\text { de análisis y } \\
\text { síntesis. }\end{array}$ & $\begin{array}{l}4,503,63 \\
4,463,48\end{array}$ \\
\hline Media glo & & $4,813,47$ & & $4,483,56$ \\
\hline
\end{tabular}


La Tabla 6 muestra los promedios de la dimensión técnico-profesional. Las valoraciones de la importancia de las competencias generales y transversales son también altas y próximas $(\bar{X}=4.78$ y $\bar{X}=4.62)$, si bien las competencias transversales son nuevamente las que reciben menor puntuación. La valoración de la adquisición de ambos tipos de competencias es también muy inferior a la importancia, $\bar{X}=3.11$ y $\bar{X}=2.88$ respectivamente. Destaca la circunstancia de que la adquisición de las competencias transversales de esta dimensión se sitúa por debajo del valor central de la escala.

\section{Tabla 6. Promedios de la dimensión técnico-profesional.}

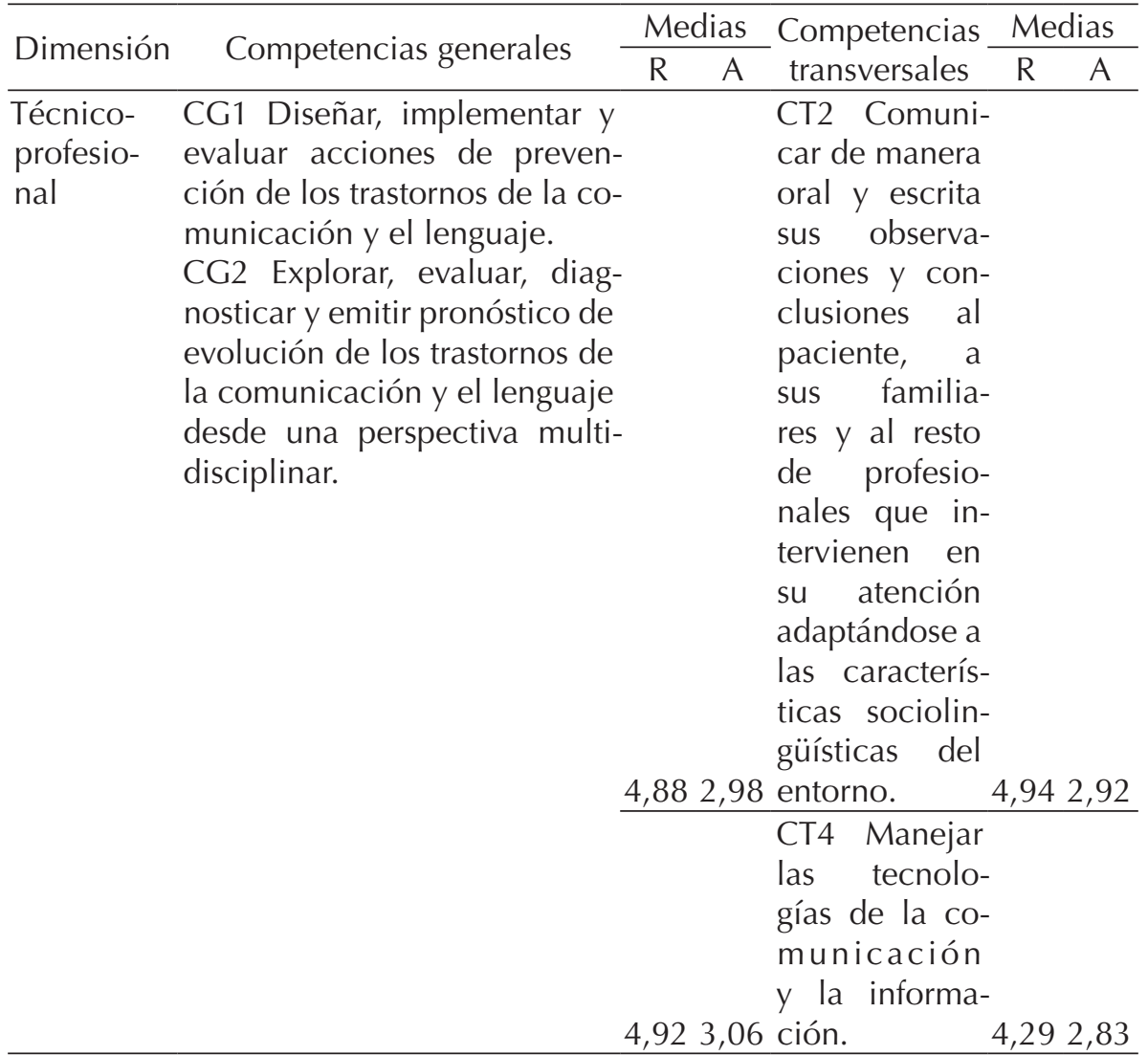


Las competencias generales y transversales del Grado en Logopedia desde la perspectiva del alumno

Manuel Rodríguez Sánchez y Pilar Revilla Rodríguez

\begin{tabular}{|c|c|c|c|c|c|}
\hline \multirow{2}{*}{ Dimensión } & \multirow{2}{*}{ Competencias generales } & Medias & \multirow{2}{*}{$\begin{array}{c}\text { Competencias } \\
\text { transversales }\end{array}$} & \multicolumn{2}{|c|}{ Medias } \\
\hline & & $A$ & & $\mathrm{R}$ & A \\
\hline & $\begin{array}{l}\text { CG3 Usar las técnicas e ins- } \\
\text { trumentos de exploración pro- } \\
\text { pios de la profesión y registrar, } \\
\text { sintetizar e interpretar los da- } \\
\text { tos aportados integrándolos en } \\
\text { el conjunto de la información. }\end{array}$ & $4,883,08$ & & & \\
\hline & $\begin{array}{l}\text { CG4 Dominar la terminología } \\
\text { que permita interactuar eficaz- } \\
\text { mente con otros profesionales. }\end{array}$ & $4,813,96$ & & & \\
\hline & $\begin{array}{l}\text { CG5 Diseñar y llevar a cabo } \\
\text { los tratamientos logopédicos, } \\
\text { tanto individuales como co- } \\
\text { lectivos, estableciendo objeti- } \\
\text { vos y etapas, con los métodos, } \\
\text { técnicas y recursos más efica- } \\
\text { ces y adecuados, y atendien- } \\
\text { do a las diferentes etapas evo- } \\
\text { lutivas del ser humano. }\end{array}$ & $4,98 \quad 2,88$ & & & \\
\hline
\end{tabular}

CG6 Seleccionar, implementar y facilitar el aprendizaje de sistemas de comunicación aumentativos, así como el diseño y uso de prótesis y ayudas técnicas necesarias, adaptados a las condiciones físicas, psicológicas y sociales de sus pacientes. 4,77 3,23

CG7 Asesorar a familias y al entorno social de los usuarios, favoreciendo su participación y colaboración en el tratamiento logopédico.

CG12 Fomentar las habilidades comunicativas en la población general. $4,002,98$

CG17 Elaborar y redactar informes de exploración y diagnóstico, seguimiento, finalización y derivación. $4,982,60$ 
La Tabla 7 recoge los promedios de las competencias incluidas en la dimensión personal. Los promedios de conjunto de las competencias transversales son análogos a los de las dimensiones anteriores, siendo mayor el de la importancia $(\bar{X}=4.63)$ y sensiblemente inferior el de la adquisición ( $\bar{X}=3.58$ ). El Gráfico 2 sintetiza las puntuaciones medias de las tres dimensiones por tipo de competencia, importancia y adquisición.

Tabla 7. Promedios de la dimensión personal.

\begin{tabular}{llll}
\hline \multirow{2}{*}{ Dimensión } & \multicolumn{2}{l}{ Medias } \\
\cline { 3 - 4 } $\begin{array}{l}\text { Personal } \\
\text { (actitudes }\end{array}$ & CT1 Ser capaz de desarrollar habilidades como: & & \\
y valores) & CT1A regular su propio & & \\
& $\quad$ Aprendizaje. & 4,35 & 3,58 \\
& CT1B resolver problemas. & 4,60 & 3,19 \\
& CT1C razonar críticamente. & 4,60 & 3,29 \\
& CT1D adaptarse a situaciones & 4,60 & 3,50 \\
& $\quad$ Nuevas. & 4,88 & 3,10 \\
& CT3 Presentar una adecuada producción del habla, & & \\
& estructuración del lenguaje y calidad de la voz. & 4,75 & 4,02 \\
& CT5 Observar y escuchar activamente. & 4,65 & 4,40 \\
& CT6 Trabajar en equipo. & & \\
\hline
\end{tabular}

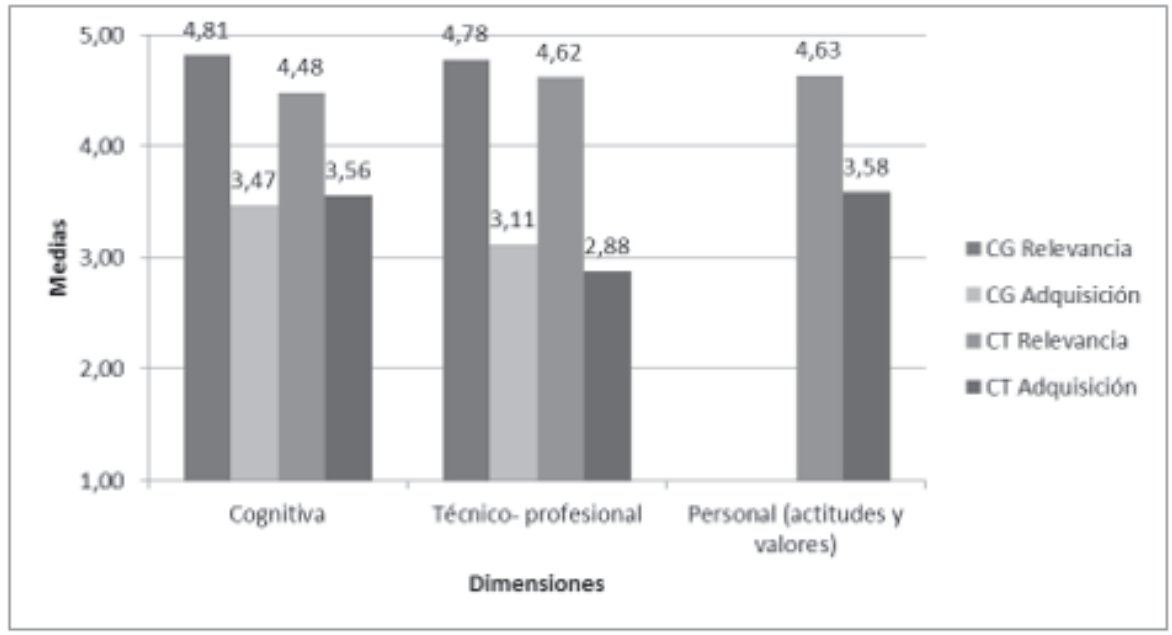

Gráfico 2. Promedios por dimensiones. 
Las competencias generales y transversales del Grado en Logopedia desde la perspectiva del alumno

Manuel Rodríguez Sánchez y Pilar Revilla Rodríguez

\section{Discusión y conclusiones}

Los resultados muestran que en todas las competencias, tanto transversales como generales, los alumnos perciben la adquisición de competencias en menor grado que la relevancia que les conceden, consideran relevantes o muy relevantes las competencias descritas para el Titulo de Grado, tanto transversales como generales, pero son más conservadores en la consideración de su propia adquisición. Es destacable que en todas las competencias se mantenga el sentido de la diferencia y que sólo en dos competencias -trabajo en equipo y observación y escucha activas-, ambas transversales y de dimensión personal, las medias se aproximen y que sólo en la primera de estas dos la diferencia no resulte significativa.

La alta valoración concedida a la relevancia de las competencias lleva a concluir que, en la percepción de los alumnos, la estructura competencial del Título está bien construida y que el currículum que de ella se deriva es consistente con el perfil profesional esperado. Aún asumiendo la lógica de que los alumnos se encuentren inseguros en sus competencias profesionales en los momentos últimos de su formación, dada su inexperiencia y escaso conocimiento del mundo laboral y profesional que sólo les ha sido mostrado breve y parcialmente en los periodos de prácticas externas, son destacables las bajas valoraciones concedidas a su propia adquisición competencial, que en varios casos se sitúa por debajo del valor central de la escala, en mayor medida en las competencias generales, más orientadas al ejercicio profesional, que en las transversales, más orientadas al desarrollo de habilidades comunes a la mayor parte de las titulaciones universitarias.

Las mayores diferencias entre lo considerado relevante y lo adquirido se observan en las tres competencias generales Diseñar y llevar a cabo tratamientos, Trabajar en entornos escolares, asistencial y sanitario y Elaborar y redactar informes, todas ellas fundamentales para la práctica profesional y con diferencias mayores a dos puntos de la escala, siendo la mayor diferencia la observada en Elaborar y redactar informes, competencia que resulta una de las más valoradas $(\bar{X}=4.98)$ en su importancia pero con menor percepción del grado de adquisición $(\bar{X}=2.60)$. La conclusión que de ello se desprende es que los alumnos perciben que la formación que han recibido se acerca más al conocimiento teórico que al hacer práctico

Considerando las dimensiones, las puntuaciones más altas en cuanto 
al reconocimiento de su importancia las recibe la dimensión cognitiva, con media global de $\bar{X}=4.81$ seguida a muy escasa distancia de la dimensión técnico profesional, con media global de $\bar{X}=4.78$. Se observa que las competencias cuya importancia es considerada en mayor grado son, paralelamente, las que reciben peores puntuaciones en cuanto a al reconocimiento de su adquisición.

Por otra parte, en cuanto a las competencias adquiridas, las cognitivas reciben mayores puntuaciones que las técnico profesionales y las personales, de lo que se desprende que los alumnos se consideran mejor capacitados en el plano teórico que en el práctico, lo que es coherente con el supuesto de inexperiencia e inseguridad, y por ello desde las instancias académicas debe ser tenido en cuenta y ser objeto de estudio y consideración a fin de que los titulados egresados lo hagan con mayor capacitación profesional, que sólo puede obtenerse desde la experiencia y la práctica.

\section{Referencias bibliográficas}

Barnett, R. (2001). Los límites de la competencia. El conocimiento, la educación superior y la sociedad. Barcelona: Gedisa.

Bisquerra, R. (2004). Competencias emocionales y educación emocional. Comunicación presentada a IV Jornadas Técnicas de Orientación Profesional, Zaragoza. https://servicios.aragon.es/redo/contenido.streamFichero.do?idBinario=1201

Bunk, G. P. (1994). La transmisión de las competencias en la formación y el perfeccionamiento profesionales de la República Federal Alemana. Revista Europea de Formación Profesional, 2, 8-14.

Cebriá i Iranzo, M. A., Díaz Díaz, B., y Igual Camacho, C. (2009). Título de grado en Fisoterapia: valoración de las competencias por parte del estudiante. Fisoterapia, 32(2), 73-79.

Cela, J. M., Arias, I., y Esteve, V. (2009). COMPTIU: Competencias transversales básicas para la incorporación en la universidad. Comunicación presentada a EDUTEC 2009: sociedad del conocimiento y el medio ambiente: sinergia científica y las TIC generando desarrollo sostenible, Manaus, Brasil.

De la Iglesia Villasol, M. C. (2011). Adecuación del grado de desarrollo de la formación en competencias a la necesidad en el entorno laboral, según la opinión de los estudiantes. Revista Complutense de Educación, 22(1), 71-92.

De la Iglesia Villasol, M. C. (2012). La identificación de factores en el desarrollo de competencias de los estudiantes universitarios. Un estudio exploratorio. Revista Complutense de Educación, 23(1), 207-240.

Díez González, M. C., Pacheco, D. I., García Saánchez, J. N., Martínez Cocó, B., Roble- 
Las competencias generales y transversales del Grado en Logopedia desde la perspectiva del alumno

Manuel Rodríguez Sánchez y Pilar Revilla Rodríguez

do Ramón, P., Álvarez Fernández, M. L., et al. (2009). Percepción de los estudiantes universitarios de educación respecto al uso de metodologías docentes y el desarrollo de competencias ante la adaptación al EEES: datos de la Universidad de Valladolid. Aula Abierta, 37(1), 45-56.

Echeverría, B. (2001). Configuración actual de la profesionalidad. Letras de Deusto, 31, 35-55.

Echeverría, B. (2002). Gestión de la competencia de acción profesional. Revista de Investigación Educativa, 20(1), 7-43.

Gallego, C. (Ed.). (2004). Libro Blanco. Título de Grado en Logopedia. Madrid: Agencia Nacional de Evaluación de la Calidad y Acreditación (ANECA).

González, J., y Wagenaar, R. (2003). Tunning educational structures in Europe. Informe final. Deusto: Universidad de Deusto.

Le Boterf, G. (2001). Ingeniería de las competencias. Barcelona: Gestión 2000.

López Ruiz, J. I. (2011). Un giro copernicano en la enseñanza universitaria: formación por competencias. Revista de Educación, 356, 279-301.

Montoro-Sánchez, M. A., Mora-Valentín, E. M., y Ortiz-de-Urbina-Criado, M. (2012). Análisis de las competencias adquiridas en los estudios de Dirección de Empresas y su grado de aplicación en las prácticas en empresas. Revista Complutense de Educación, 23(1), 241-263.

OCDE. (2006). La definición y selección de competencias clave. Resumen ejecutivo. 1-20. Localizado en http://www.deseco.admin.ch/bfs/deseco/en/index/03/02.parsys.78532.downloadList.94248. DownloadFile.tmp/2005.dscexecutivesummary. sp.pdf

Rey, B. (1996). Les competénces transversales en question. Paris: ESF.

Rodríguez-Sánchez, M. (2011). El profesor en el aula: metodología docente orientada al desarrollo de las competencias. En S. Morales (Ed.), Nuevos contextos de enseñanza y aprendizaje en el Espacio Europeo de Enseñanza Superior (pp. 99-126). Buenos Aires: Miño y Dávila.

Romero Cerezo, C., Zagalaz Sánchez, M. L., Romero Rodríguez, M. N., y Martínez López, E. J. (2011). Importancia de las competencias profesionales de los Maestros en Educación Física expresadas por los estudiantes. Retos. Nuevas tendencias en Educación Física, Deporte y Recreación, 19, 62-68.

Valverde, O. (Ed.). (2001). El enfoque de la competencia laboral. Montevideo: Cinterfor/ OIT. 